purposively selected from a range of socio-economic backgrounds. Semi-structured interviews were completed with eleven parents (nine mothers; two fathers) in parents' homes. Transcripts were analysed using thematic analysis.

Results Two themes were developed from analysis. 'Quality of communication' describes how parents develop confidence in the SALT and open communication. 'Shared responsibility' describes how parents felt supported by and jointly responsible with the SALT in helping their child. Elements of the interaction that may help SALTs engage parents more fully are: sharing expertise freely, communicating information clearly, demonstrating understanding of the parents' perspective, treating the parent as an equal, encouraging open dialogue, supporting parents, showing genuine interest in the child and providing personalised and achievable therapy advice.

Conclusion and implications for clinical practice This study has increased understanding of how SALTs can work with parents to optimise engagement. SALTs can consider elements of the parent-SALT interaction that they may need to demonstrate more fully to engage parents with therapy. Future studies could measure and evaluate these elements.

\section{P16 CANCER SURVIVORS' EXPERIENCE WITH TELEHEALTH: A SYSTEMATIC REVIEW AND THEMATIC SYNTHESIS}

${ }^{1}$ Anna Cox, ${ }^{2}$ Grace Lucas, ${ }^{1}$ Afrodita Marcu, ${ }^{1}$ Wendy Grosvenor, ${ }^{1}$ Freda Mold, ${ }^{3}$ Roma Maguire, ${ }^{1}$ Marianne Piano, ${ }^{1}$ Emma Ream. ' ${ }^{1}$ University of Surrey, Guildford, UK; ${ }^{2}$ City University, London, UK; ${ }^{3}$ University of Strathclyde, Glasgow, UK

\subsection{6/bmjopen-2019-QHRN.51}

Background There is a drive to transfer the care of cancer survivors to the community and encourage them to play an active role in their own care. Telehealth is an important contributor to this evolving model of care but we are lacking an integrative perspective and in-depth understanding of how cancer survivors accept it, engage with it, and benefit from it.

Objective To systematically identify, appraise, and synthesize qualitative research evidence on the experiences of adult cancer survivors participating in telehealth interventions, to characterize the patient experience of telehealth interventions for this group.

Methods We searched PsycINFO, Medline, CINAHL, Embase, and Cochrane Central Register of Controlled Trials for studies published in English between 2006 and 2016 reporting qualitative data (including verbatim quotes) on adult cancer survivors' experience of participating in a telehealth care intervention (defined as remote communication or remote monitoring with a health care professional delivered by telephone, Internet, or hand-held or mobile technology). All papers underwent inductive analysis, involving comparison, reexamination, and grouping of codes to develop descriptive themes. Analytical themes were developed through an iterative process of reflection on, and interpretation of, the descriptive themes within and across studies.

Results Across 22 papers included in the review, three analytical themes emerged, each with three descriptive subthemes: (1) influence of telehealth on the disrupted lives of cancer survivors (convenience, independence, and burden); (2) personalized care across physical distance (time, space, and the human factor); and (3) remote reassurance-a safety net of health care professional connection (active connection, passive connection, and slipping through the net). Telehealth interventions represent a convenient approach to cancer care, which can potentially minimise treatment burden and disruption to cancer survivors' lives.

Conclusions Telehealth interventions can engender an experience of personalised care, while providing cancer survivors with independence and reassurance.

\section{P17 ADOPTING THE NOMINAL GROUP TECHNIQUE TO UNDERSTAND THE USE OF RELIGIOUS PRACTICES AND EXPERIENCES}

${ }^{1}$ Ophelia Anarfi, ${ }^{2}$ Stella Dzifa Monu, ${ }^{1,3}$ Charles Christopher Mate-Kole. ${ }^{1}$ Department of Psychology, University of Ghana-Legon, Accra-Greater Accra Region, Ghana; ${ }^{2}$ Korle-Bu Teaching Hospital, Accra, Ghana; ${ }^{3}$ Centre for Aging Studies, University of Ghana, AccraLegon, Ghana

\subsection{6/bmjopen-2019-QHRN.52}

Background Religion plays a vital role in recovery of illness of which stroke is not an exception.

Aims/Objectives To determine the various and use of religious experiences and practices adopted as a recovery strategy in the face of stroke condition.

Methods 20 participants would be recruited from the Stroke Unit and the Physiotherapy Unit of the Korle-Bu Teaching Hospital (KBTH to participate in two Nominal Group Technique (NGT) meeting. Stroke survivors from the first group would be asked to generate list of religious practices and experiences in response to the question 'What are the religious practices, values and beliefs that are related to the culture which aids in recovery after stroke?'; and the second group would be asked to generate responses relating to the impact of religion 'What are the positive and negative impact of religion to health and recovery after stroke?'

Expected results It should be expected that the topmost religious practices and experiences that relate to recovery after stroke would include reading of scriptures and recitation of mantra, prayers and fasting, consultation and directions from pastors, and meditation. The topmost impact of religion on recovery may include issues with adherence to treatment, resorting to alternative forms of treatment, and healershopping.

Conclusion Most of the religious practices and experiences were considered to aid in recovery after stroke. On the other hand, although there are positive aspects of religion on health, there are negative aspects of it that worsens the recovery after stroke.

\section{P18 DESIGNING FOR WELL-BEING: THE INFLUENCE OF A SCHOOLYARD INTERVENTION ON SUBJECTIVE WELL-BEING}

${ }^{1}$ Matluba Khan, ${ }^{2}$ Sarah McGeown. ${ }^{1}$ University College London, London, UK; ${ }^{2}$ University of Edinburgh, Edinburgh, UK

\subsection{6/bmjopen-2019-QHRN.53}

This study examines effects of a schoolyard intervention on primary school children's perceived motivation, exploration, peer-relation, teaching and learning in Bangladesh. $43 \%$ of the children in developing countries grow with poorer mental development. More than $21 \%$ of children leave primary 